\title{
Diabetic plantar pressure analysis using image fusion
}

Article

Accepted Version

Cao, L., Dey, N., Ashour, A. S., Fong, S., Sherratt, R. S., Wu, L. and Shi, F. (2020) Diabetic plantar pressure analysis using image fusion. Multimedia Tools and Applications, 79. pp. 11213-11236. ISSN 1573-7721 doi: https://doi.org/10.1007/s11042-018-6269-x Available at https://centaur.reading.ac.uk/77537/

It is advisable to refer to the publisher's version if you intend to cite from the work. See Guidance on citing.

To link to this article DOI: http://dx.doi.org/10.1007/s11042-018-6269-x

Publisher: Springer

All outputs in CentAUR are protected by Intellectual Property Rights law, including copyright law. Copyright and IPR is retained by the creators or other copyright holders. Terms and conditions for use of this material are defined in the End User Agreement.

\section{www.reading.ac.uk/centaur}

\section{CentAUR}

Central Archive at the University of Reading 
Reading's research outputs online 


\title{
Diabetic plantar pressure analysis using image fusion
}

\author{
Luying Cao - Nilanjan Dey • Amira \\ S. Ashour • Simon Fong · R. Simon \\ Sherratt · Lijun Wu · Fuqian Shi
}

Received: date / Accepted: date

\author{
This work is partial supported by Zhejiang Provincial Natural Science Foundation un- \\ der Grant (LY17F030014), the National Natural Science Foundation of China (Grant nos: \\ 81271663 and 31471146), Zhejiang Wenzhou Medical University Scientific Development \\ Foundation of China (Grant no: QTJ06012). \\ Luying Cao \\ College of Information and Engineering, Wenzhou Medical University, Wenzhou, 325035, \\ PR China \\ E-mail: 1294502807@qq.com \\ Nilanjan Dey \\ Dept. of IT, Techno India College of Technology, West Bengal, 740000,India \\ E-mail: nilanjan.dey@tict.edu.in \\ Amira S. Ashour \\ Department of Electronics and Electrical Communications Engineering, Faculty of Engi- \\ neering, Tanta University, Tanta 31527 Egypt \\ E-mail: amirasashour@yahoo.com \\ Simon Fong \\ Department of Computer and Information Science, University of Macau, Av. Padre Tomas \\ Pereira, Taipa, Macau SAR \\ E-mail: ccfong@umac.mo \\ R. Simon Sherratt \\ Department of Biomedical Engineering, the University of Reading, RG6 6AY, UK \\ E-mail: r.s.sherratt@reading.ac.uk \\ Lijun $\mathrm{Wu}$ \\ Institute of Digitized Medicine, Wenzhou Medical University, Wenzhou, 325035, PR China \\ Tel.: +86-577-86688659 \\ Fax: +86-577-86689922 \\ E-mail: biomech@163.com \\ Fuqian Shi \\ College of Information and Engineering, Wenzhou Medical University, Wenzhou, 325035, \\ PR China \\ Tel.: +86-577-86689913 \\ Fax: +86-577-86689222 \\ E-mail: sfq@wmu.edu.cn
}




\begin{abstract}
Plantar pressure images analysis is the key issue of designing comfortable shoe products through last customizing system, which has attracted the researchers' curiosity toward image fusion as an application of medical and industrial imaging. In the current work, image fusion has been applied using wavelet transform and compared with Laplace Pyramid. Using image fusion rules of Mean-Max, we presented a plantar pressure image fusion method employing haar wavelet transform. It was compared in different composition layers with the Laplace pyramid transform. The experimental studies deployed the haar, db2, sym4, coif2, and bior5.5 wavelet basis functions for image fusion under decomposition layers of 3,4 , and 5 . Evaluation metrics were measured in the case of the different layer number of wavelet decomposition to determine the best decomposition level and to evaluate the fused image quality using with different wavelet functions. The best wavelet basis function and decomposition layers were selected through the analysis and the evaluation measurements. This study established that haar wavelet transform with five decomposition levels on plantar pressure image achieved superior performance of $89.2817 \%$ mean, $89.4913 \%$ standard deviation, 5.4196 average gradient, 14.3364 spatial frequency, 5.9323 information entropy and 0.2206 cross entropy.
\end{abstract}

Keywords biomedical signal processing · image fusion · plantar pressure sensors · wavelet transforms · Gaussian Laplace Pyramid

\title{
1 Introduction
}

Plantar pressure distribution has a significant role in clinical medicine, medical rehabilitation assessment, sports training, biomechanics application, and shoe industry [1]. Many scholars focused on plantar pressure distribution in solving the problem on how to design a comfortable shoe. Furthermore, it can be combined with the last shoes design to effectively reduce the occurrence of foot disease. This has important significance to improve the quality of peoples life [2], [3], [4]. Several artificial intelligences, data mining, and gait studies have been applied for plantar pressure data feature extraction. Panagiotis et al. [5] developed a new magnetic resonance imaging (MRI) compatible loading device on plantar pressure distribution for foot biomechanics research. Forefoot pain based on plantar pressure measurements for clinical evaluation [6] and gait analysis [7] were also introduced. High plantar pressures have been associated with foot ulceration with diabetes. Craig et al. [8] elevated plantar pressure distributions and used k-means clustering analysis to identify typical regional peak plantar pressure distributions in a group of 819 diabetic feet. This approach provided an understanding of the variability of the regional peak plantar pressure distributions of diabetic patients. Moreover, diabetic peripheral neuropathy has been associated with plantar pressure [9]. Applying finite element model and peak pressures reducing for therapeutic insole design $[10]$ is also regarding as a research hot-spot. Since image processing based image fusion can be applied for furthermore effective information. Recently, 
researchers are interested to employ image fusion to obtain efficient plantar pressure distribution analysis.

Image fusion (IF) refers to the image data collected on the same target by multi-source channel through image processing and computer technology;it is to maximize the extraction of favorable information in their respective channels, and finally integrate into high quality images. Reliability and the enhancement of the spatial resolution and spectral resolution of the original image are easier through improving the utilization of image information and the accuracy of computer interpretation. The fused image has been registered and the pixel width is consistent.Two or more multi-source image information is synthesized and extracted. As a branch of information fusion, image fusion is a hot spot in the current information fusion research. The data form of image fusion is an image containing light and shade, color, temperature, distance and other scenery features. These images can be given in one or more columns. Image fusion is a fusion of two or more than two image information into one image making the fused image more information, more conveniently for human observation or computer processing. The goal of image fusion is to reduce the uncertainty and redundancy of output based on the maximum combination of relevant information under the actual application target. The advantages of image fusion are obvious. It can enlarge the time and space information contained in the image, reduce the uncertainty, increase the reliability and improve the robust performance of the system. The efficient image fusion method can comprehensively deal with the information of the multisource channel according to the needs, thus effectively improve the utilization rate of the image information and the system to the target. Detect and identify the reliability and automation of the system. The aim is to synthesize the information provided by the multi band information of a single sensor or the information provided by different kind of sensors to eliminate the possible redundancy and contradiction between the multi-sensor information, so as to enhance the transparency of the information in the image, improve the accuracy, reliability and usage of the interpretation, so as to form the clarity, integrity and accuracy of the target of information description. IF based on multi-resolution analysis become the research emphasis and hot-spot [11] [12]. Image fusion is carried out for more comprehensive and accurate description of the acquired image based on multi-source image fusion. It efficiently combines the different images advantages to enhance the analysis capability. Recently, the IF is employed in medical image processing, computer vision, automatic target recognition, robots, remote sensing, and military domains in a broad application range. Some improved artificial intelligent technologies have been combined in fusion operation, such as pulse coupled neural network (PCNN) based multi-faceted adaptive image fusion algorithm [13]. Piras and Giacinto introduced content based image retrieval to overcome cheap availability of powerful cameras on smartphones [14]. In the medical image applications, Zong et al. [15] performed visual perception and objective metrics to evaluate medical image fusion based on sparse representation of classified image. Magnetic resonance imaging-transrectal ultrasound fusion on men active surveillance 
[16] and focal precision therapy [17] were also developed. Multi-focus image fusion [18], multiple kernel learning based fusion [19], and deep convolutional neural network based fusion technologies [20] are also considered recently. Overcoming the limitations and differences in the single sensor image is the key feature of the IF, which is to improve the clarity and intelligibility of geometry, spectral and spatial resolution of the image.

IF can be divided into two categories, namely image fusion based on spatial domain and transform domain. Generally, multi-resolution based image fusion techniques can be categorized into three main types, namely i) IF based on wavelet decomposition including multi-wavelet transform, discrete wavelet transform, integer wavelet transform and dual tree complex wavelet transform, ii) IF based on the pyramid decomposition including ratio low-pass pyramid, gradient pyramid, Laplace pyramid and contrast pyramid, and iii) new multiresolution approaches including IF based on curvelet transform, contourlet and finite ridgelet transform. In the multi-resolution fusion process, the operators/rules selection is crucial as it affects the IF quality. The fusion rule is the core of the image fusion algorithm, which directly determines the final fusion effect. Different fusion rules "Mean-Max" can be applied to the high frequency and low frequency information, thus the low frequency and high frequency parts of the multi-scale decomposition have different physical meanings. The low frequency information usually adopts the simple average method that can effectively suppress the average noise of an image. The images low frequency component includes the main energy of the image. High frequency information fusion is possible to extract detail information of the source images characteristics, such as straight line, curve, and contour. It is often expressed as the gray value and its changes in the multi-scale transform domain often are manifested in high frequency sub-band coefficients of larger modulus. Therefore, the Mean-Max fusion rule is often used to extract the detail information of the source image in this research.

Typically, wavelet transform plays an important role in image fusion. Image cryptographic algorithm based on the haar wavelet transform [21], solution of nonlinear damped Van der Pol equation [22], remote sensing image Retrieval [23], and Electrocardiography (ECG) image classification [24] have been achieved high performance for applying wavelet transform. The suitable wavelet bases can be selected correctly based on the time-frequency characteristics analysis of wavelet bases and the image fusion technique. However, the basis function of wavelet transform is not unique. Thus, in wavelet transform based image fusion, the wavelet coefficients of each scale of the wavelet transform select wavelet basis function and have a certain extent effect on the distribution of the wavelet coefficients, hence, the wavelet directly affects the fusion quality. Wavelet bases have five important indicators, namely orthogonality, compact support, vanishing distance, regularity, and symmetry. In addition, the general wavelet basis functions are haar, db2, coif2, sym4, and bior5.5.

Recently, multi-scale image fusion is considered a mainstream method of wavelet transform based image fusion. The technologies of wavelet transform 
based image fusion include Pyramid transform, which is the most classical multi-scale decomposition method that is widely used in IF. Laplace Pyramid transform based image fusion has a wide range of features. It produces only a band-pass signal at each level, so that it can be applied in many applications from coarse to fine multi-resolution algorithm under superior treatment effect. Laplace Pyramid decomposition based IF divides the image into different scales and resolutions, the fusion of different scales and spatial frequencies and decomposition layer respectively can significantly improve the fusion effect.

Haar feature for recognizing achieved significant results as reported by Zheng et al. [25] to study the fatigue state of the vehicle driver to avoid the traffic accident. In addition, Humberto et al. estimated the non-subsampled Haar wavelet coefficients by using a linear estimator for high-resolution small animal 3D positron emission tomography (PET) data fusion [26]. Furthermore, the fractional Fourier (FRT) and linear canonical transforms (LCT) [27], and Walsh-hadamard transform [28] have been developed based on Haar wavelet transform. Consequently, the current work applied wavelet basis functions of haar for plantar pressure image fusion operation. Low frequency averaging and maximum frequency of fusion rules are applied in the present work. Evaluation indexes including mean, standard deviation, the information entropy, average gradient, spatial frequency, and mean cross entropy are also calculated. Cross entropy is an important concept in Shannon information theory. It is mainly used to measure the difference information between two probability distributions. The performance of language models is usually measured by cross entropy and complexity (perplexity). The meaning of cross entropy is the difficulty of text recognition with the model, or from the compression point of view, each word is encoded in several bits on average. The meaning of complexity is to use this model to represent the average number of branches of the text, and the reciprocal can be regarded as the average probability of each word. Smoothing refers to giving a probability value to the $\mathrm{N}$ element combination that is not observed, so as to ensure that the word sequence can always get a probability value through the language model. The smoothing techniques commonly used include Turing estimation, interpolation smoothing, Katz smoothing and Kneser-Ney smoothing. Applying artificial (AI) intelligent technologies in medical images mining filed has been a hot-spot topic including protein structure [29], gene codding virilization [30] and meta-genomic data classification [31][32]. Those AI technologies mentioned can be used in plantar pressure imaging data-set which can further promote and deep explore the diabetic plantar pressure research. In addition, the wavelet fusion and Laplace Pyramid transform with different wavelet bases functions are discussed.

\section{Methods}

In the current work, plantar pressure images fusion is proposed to extract the significant features. Image fusion rule of Mean-Max is applied. In addition, haar wavelet transform is used and is compared in different composition layers 
of Laplace Pyramid transform. Afterward, haar, db2, sym4, coif2, and bior5.5 wavelet basis functions which are typical for image fusion issue, are carried out under different decomposition layers of 3,4 , and 5 . The superior wavelet basis function and decomposition layers are selected by analyzing and comparing the different wavelet based image fusion results. The proposed framework of plantar pressure image fusion and evaluation is demonstrated in Fig. 1. In re-

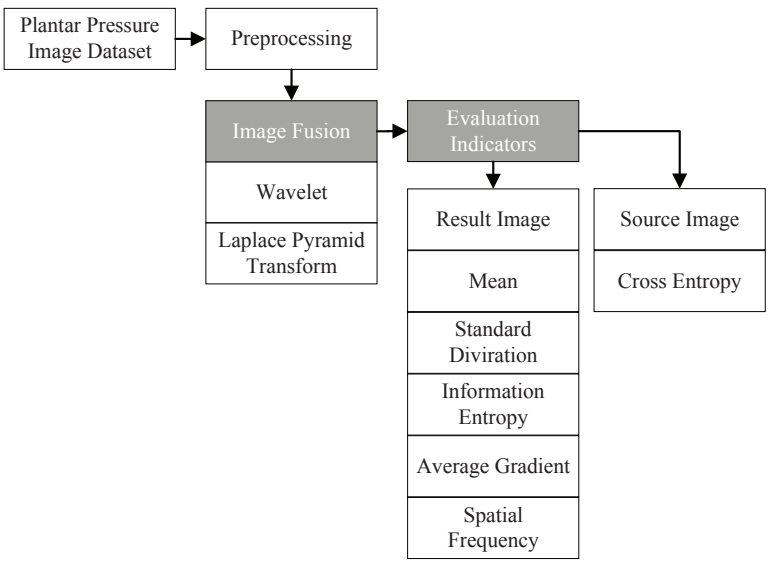

Fig. 1 Proposed framework of plantar pressure image fusion and evaluation

sults image evaluation issue, some indices were introduced by previous studies for image fusion. Mean (M) is a typical linear filtering algorithm, which refers to a template on the target pixel in the image, it includes the adjacent pixels around the target ( 8 pixels around the target pixel, forming a filter template, that is, to remove the target pixel itself), and then use the average value of all the pixels in the template to replace it. Standard Derivation (SD) is the square root of the arithmetic mean of the square of the homodyne. Information Entropy (E) is the meaning of information entropy of the image.It characterizes the aggregation characteristics of the gray distribution of the image. In order to characterize this spatial feature, it can introduce the two-dimensional entropy which can reflect the feature quantity of the gray distribution spatial feature to form the image on the basis of the one-dimensional entropy. Entropy is represented as the bit average of the set of image gray level, and the unit bit / pixel also describes the average information content of the image source. Average gradient (AG) is the edge of the image or the gray level of the two different sides of the film, that is, the gray change rate is large, and the size of the change rate can be used to express the image definition. It reflects the rate of contrast variation of image minutiae, that is, the rate of density change in multidimensional direction of the image, which represents the relative clearness of the image. Spatial frequency (SF) refers to the number of bars per sinusoid modulated by the bright or the dark shape of the image in 
each view. It is based on the theory of vibration wave and describes the concept of working characteristics of the vision system. In physical optics, space frequency refers to the number of grating per millimeter per unit.

\subsection{Image Fusion with Haar Transform}

Multi-resolution analysis of wavelet transform is the hotspot of information and image processing domain. Mallat et al. established the relationship between the multi-resolution analysis and wavelet analysis to obtain a fastdiscrete wavelet transform algorithm, namely Mallat algorithm [33]. Typically, the original image wavelet transform is decomposed into a series of different spatial resolution and frequency features of sub-images. It can fully reflect the localization characteristics of the original image, which provides favorable conditions for image data fusion. In wavelet transform, for a function $\psi \in L^{2} \mid L^{1}$, $a, b \in R$, the analytic wavelet (continuous wavelet) family function can be generated as follows:

$$
\psi_{a, b}(t)=|a|^{-\frac{1}{2}} \psi\left(\frac{t-b}{a}\right)
$$

where, t means time, $\psi(t)$ is the basic wavelet, $a$ is a scale parameter and $b$ is a translation parameter. The extension and contraction function of $\psi_{a, b}(t)$ are relative to $a$ and translation function of $\psi_{a, b}(t)$ are relative to $b$. The choice of $\psi(t)$ is not unique and arbitrary. Typically, the analytic function $\psi(t)$ has unit energy normalization under two conditions, namely i) the domain is compactly supported, i.e., in a very small range, as a function of zero, the function feature need to be downhill; and ii) the average value is zero. These two conditions indicate that the wavelet should be an oscillatory and rapidly decaying wave. Thus, the wavelet transform on a continuous function $f$ is expressed as follow:

$$
w_{f}(a, b)=\int_{-\infty}^{+\infty} f(t) \psi_{a, b}(t) d t=\frac{1}{\sqrt{a}} \int_{-\infty}^{+\infty} f(t) \psi\left(\frac{t-b}{a}\right) d t
$$

Consequently, the inverse transform is given by:

$$
f(t)=\frac{1}{c_{\psi}} \int_{-\infty}^{+\infty} \int_{-\infty}^{+\infty} a^{-2} w_{f}(a, b) \psi_{a, b}(t) d a d b
$$

where, $c_{\psi}=\int_{-\infty}^{+\infty} \frac{\psi(\varpi)^{2}}{\varpi} d \varpi<\infty$ as

$$
\psi(\varpi)=\int_{-\infty}^{+\infty} \psi(t) e^{-j w t} d t
$$

In the process of image fusion, the fusion rules and the fusion operators selection are significantly affecting the fusion quality. The basic steps of wavelet transform based plantar pressure image fusion are:

- The wavelet transform of the plantar pressure image is decomposed by wavelet decomposition, and Laplace Pyramid; 
- Each decomposition layer is fused respectively. In addition, according to the different frequency components characteristics, the decomposed wavelet coefficients can adopt different fusion strategies (fusion rules and fusion operator);

- Wavelet transform is used to reconstruct the fused wavelet Pyramid.

The flowchart of wavelet transform based plantar pressure image fusion is illustrated in Fig. 2. Image $A$ and Image $B$ are source images and plantar pressure images of the same person at different times.

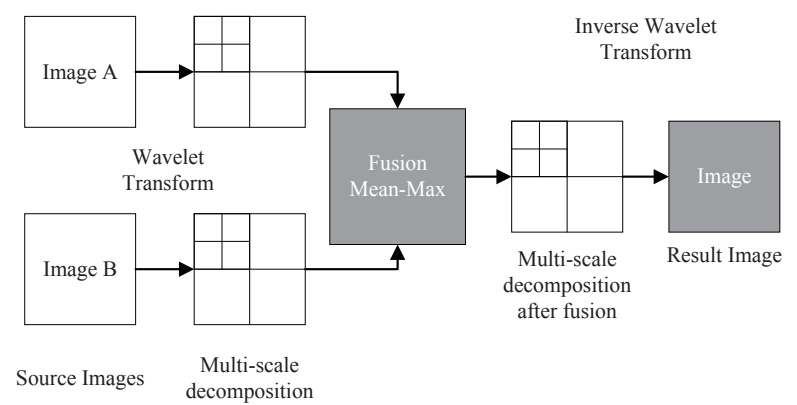

Fig. 2 The flowchart of wavelet transform based plantar pressure image fusion

\subsection{Laplacian Pyramid Transform}

\subsubsection{Gaussian Pyramid Decomposition}

The first step is the decomposition on the target image using Gaussian Pyramid, where the image of Laplace Pyramid is formed by Gaussian Pyramid. Assume the original image is represented by $G_{0}$, hence $G_{0}$ is assigned as the $0^{\text {th }}$ layer of the Gaussian Pyramid transform. The first layer is the Gauss lowpass filter and interlaced row sampling on the original input image. Afterward, the image low-pass filtering and down-sampling is the second layer of Gaussian Pyramid. This process is repeated and formalized as follows:

$$
G_{l}(i, j)=\sum_{m=-2}^{2} \sum_{n=-2}^{2} w(m, n) G_{l-1}(2 i+m, 2 j+n)
$$

where, $1 \leq l \leq N, 0<i<c_{l}, 0<j<R_{l}$ and $N$ is the layer number of Gaussian Pyramid, $C_{l}$ and $R_{l}$ are the $l^{t h}$ layers column and volume; respectively, 
and $w(m, n)$ is the separated window function of $5 \times 5$, which is given by:

$$
w=\frac{1}{256}\left[\begin{array}{lllll}
1 & 4 & 6 & 4 & 1 \\
4 & 16 & 24 & 16 & 4 \\
6 & 24 & 36 & 24 & 6 \\
4 & 16 & 24 & 16 & 4 \\
1 & 4 & 6 & 4 & 1
\end{array}\right]
$$

The constructed Gaussian Pyramid is given by $G_{0}, G_{1}, \cdot, G_{n}$ and the size of current layers image is one fourth of previous layers.

\subsubsection{Laplace Pyramid Decomposition}

To find the Laplace pyramid decomposition of an image, the interpolation is performed on $G_{l}$ to image $G_{l}^{*}$, and makes it with the same size as follows:

$$
G_{l}^{*}(i, j)=4 \sum_{m=-2}^{2} \sum_{n=-2}^{2} w(m, n) G_{l}^{\prime}\left(\frac{m+i}{2}, \frac{n+j}{2}\right)
$$

where, $1 \leq l \leq N, 0<i<c_{l}, 0<j<R_{l}$, and $L P_{l}$ is the layers image of Laplace Pyramid, thus,

$$
L P_{l}=G_{l}-G_{l+1}^{*}
$$

where, $0 \leq l \leq N, L P_{N}=G_{N}, l=N$ and $L P_{0}, L P_{1}, \cdot L P_{N}$ are consist of Laplace Pyramid. Each layer of the image is the difference between the image of Gauss Pyramid and the high level of the image magnified by the amplification operator.

\subsubsection{Laplace Pyramid Reconstruction}

For Laplace pyramid reconstruction of an image, the following expression is applied.

$$
G_{l}=L P_{l}+G_{l}^{*}
$$

where, $0 \leq l \leq N$. The corresponding Pyramid can be acquired by recursive from the top layer of the Laplace pyramid, and finally original image can be generated. The basic steps of Laplace pyramid transform based image fusion are as follows:

- Each source image is decomposed by Laplacian, and the Laplace pyramid of each image is established.

- Each decomposition layer of the image is fused separately. Different decomposition layers are fused with different fusion operators, and finally the fused image of Laplacian pyramid is obtained.

- The fused image is reconstructed by inverse tower type decomposition (i.e. image reconstruction).

To evaluate the performance of this proposed fusion process by Mean-Max, the following evaluation metrics are measured. 


\subsection{Fused Image Quality Evaluation}

Fusion result evaluation is another topic in image fusion operation, where several metrics can be calculated. Oliva et al. [34] used cross entropy for evaluating thresholding based segmenting operation on brain MRI dataset. Qian et al. [35] introduced deep gradient information (DGI) based on histogram of oriented gradient for biometric image feature representation. Furthermore, Zhou et al. [36] introduced local gradient patterns for full-reference image quality assessment. Mainly, the image fusion evaluation methods include two categories, namely subjective evaluation and objective evaluation. Subjective evaluation is related to the personnel involved in the evaluation. However, to overcome the influence of subjective factors, an objective evaluation is performed using quantitative evaluation method and fusion criterion to carry out more in-depth study. The evaluation performance indexes (metrics) of different fusion images have different characteristics, and the selection of fusion image performance evaluation index is based on the purpose of image fusion. Since the current work focused on image fusion of plantar pressure images, the choice of evaluation index should measure the clarity of the fused image and the amount of information and rich layers. Thus, in the current work, in the case of wavelet decomposition layer number, to determine and to combine with image fusion evaluation method, the mean (M), standard deviation (SD), information entropy (E), average gradient (AG), spatial frequency $(\mathrm{SF})$, and mean cross entropy (MCE) are calculated for evaluating the fused plantar image quality and to be compared to different wavelet functions. These statistics measurements based evaluation of the fused image are defined as follows.

\subsubsection{Mean}

It denotes the image average illumination and positive contributed the fusion results, which can be presented by:

$$
M=\frac{1}{M N} \sum_{i=1}^{N} \sum_{j=1}^{M} F(i, j)
$$

where, $F(i, j)$ is pixel of point $(i, j)$ and $M \times N$ is the size of image $F$.

\subsubsection{Standard Deviation}

It reflects the discrete values of the gray value of the image with respect to the average value of gray level. If the standard deviation is large, the image gray level distribution is scattered, the contrast of the image is large, and more information can be involved, which can be expressed as follows:

$$
S D=\sqrt{\frac{1}{M N} \sum_{i=1}^{N} \sum_{j=1}^{M}(F(i, j)-M)}
$$




\subsubsection{Image Entropy}

It is an important index to measure the richness of image information, which can be expressed as:

$$
E=-\sum_{i=0}^{L-1} P_{i} \log _{2} P_{i}
$$

where, $L$ presents amounts of gray levels and $P(i)$ presents the probability of $i$ gray value in pixels of image. The greater the entropy of the image, the greater the amount of information contained in the image, the richer the information contained in the fusion image, the better the quality of fusion.

\subsubsection{Average Gradient}

It is an index reflecting the sharpness of the image and the ability to express the small details in the image and the texture change. The higher the average gradient, the richer the image gray scale, it can be expressed as:

$$
\begin{gathered}
A G= \\
\frac{1}{(M-1)(N-1)} \sum_{i=1}^{M-1} \sum_{j=1}^{N-1} \sqrt{\frac{(F(i, j)-F(i+1, j))^{2}+(F(i, j)-F(i, j+1))^{2}}{2}}
\end{gathered}
$$

\subsubsection{Spatial Frequency}

It reflects the overall activity of an image spatial domain, which is an important index to measure the detail information of the image including the spatial frequency $R F$ and the spatial frequency $C F$, which are defined as:

$$
\begin{gathered}
R F=\frac{1}{N M} \sum_{i=1}^{N} \sum_{j=2}^{M}[I(i, j)-I(i, j-1)]^{2} \\
C F=\frac{1}{N M} \sum_{j=1}^{N} \sum_{i=2}^{M}[I(i, j)-I(i-1, j)]^{2} \\
S F=\sqrt{R F^{2}+C F^{2}}
\end{gathered}
$$

The fused image will have superior fusion affect with the increase in the spatial frequency, the richer the image level, and the increased contrast.

Furthermore, the evaluation indicator of mean cross entropy (MCE) for calculating the difference between fused image and one of source image can be given as 


\subsubsection{Mean Cross Entropy}

It is used to measure the difference between the fused image and the source image gray level distribution. The two source images are $A$ and $B$, and the fused image is $F$. Can be expressed as:

$$
M C E=\frac{1}{2}\left(\sum_{i=0}^{L-1} P_{A_{i}} \log _{2} \frac{P_{A_{i}}}{P_{F_{i}}}+\sum_{i=0}^{L-1} P_{B_{i}} \log _{2} \frac{P_{B_{i}}}{P_{F_{i}}}\right)
$$

The MCE reflects the difference of the corresponding pixels of the two images, the smaller the MCE, the smaller the difference between the fused image and the source image.

\subsection{Plantar Pressure Dataset}

Current medical research shows that there are significant differences in plantar pressure, impulse and time between diabetic patients and normal people. In particular, plantar pressure is more obvious in type-II diabetic patients. The factors affecting the plantar pressure of diabetic patients include neuropathy, joint mobility, foot deformity, callosal and so on. The key to prevent diabetic foot is to measure the patient's plantar pressure correctly and to take measures to influence the increase of plantar pressure on the basis of the analysis of the plantar pressure parameters and distribution of the diabetic patients. In general, a large number of cases are used to compare the plantar pressure of the natural walking process of type-II diabetes patients to compare the diabetic patients with the Footscan pressure plate produced by the RS-SCAN system. The acquisition equipment of the plantar pressure image acquisition is a three-dimensional time sequence information of the gait pressure image, which first extracts and expresses the three-dimensional information into two-dimensional feature information through the features. Secondly, the two-dimensional features of the subjects are linear sparse representation of the training set. Each category restores the two-dimensional information represented by this category through its own sparsity coefficient. The class of the two-dimensional difference between the actual two-dimensional features and the recovered two-dimensional features can be regarded as the category of the tested person. The plantar pressure image is also a common image, which has the characteristics of color, texture, shape and spatial relations. And feature is the best way to describe the pattern. We usually think that each dimension of the feature can describe the pattern from different aspects. In the ideal case, the dimensions are complementary and complete. The main purpose of feature extraction is to reduce the dimension and the main idea of feature extraction is to get projection the original sample to a low dimensional feature space and the characteristics of the low dimensional sample which can most respond to the essence of the sample or distinguish the sample.

To evaluate the proposed fusion system, image construction is done using a Foot-Scans 7.0 of the Rsscan system to collect the plantar pressure image 
Table 1 The performance of the scan system

\begin{tabular}{llll}
\hline Item & Value & Item & Value \\
\hline Area & $40^{*} 50 \mathrm{~cm}^{2}$ & Total sensors & 4096 \\
Sensors & 4 per $\mathrm{cm}^{2}$ & Sampling rate & $125-300 \mathrm{~Hz}$ \\
Each sensor size & $0.5 \times 0.7 \mathrm{~cm}^{2}$ & Analog channels & 16 \\
Image resolution & $12 \mathrm{bits}$ & Entry level & $2 \mathrm{~m}$ \\
\hline
\end{tabular}

data from a total of 10 diabetic patients (II type) during 20 minutes test for each student. Table 1 reported the parameters of the used scan system.

The output of the pressure scan system provided the pressure information under the foot during unroll of the foot. This system pressure outputs are based on the automatic calculated zones and the localized small spots under the foot as illustrated in Fig. 3. The $y$-axis of the figure is pressure $(N / \mathrm{cm})$, and $\mathrm{x}$-axis means time $(s)$, the colored lines are different zone of foot. The left sub figures present colored pressure values.

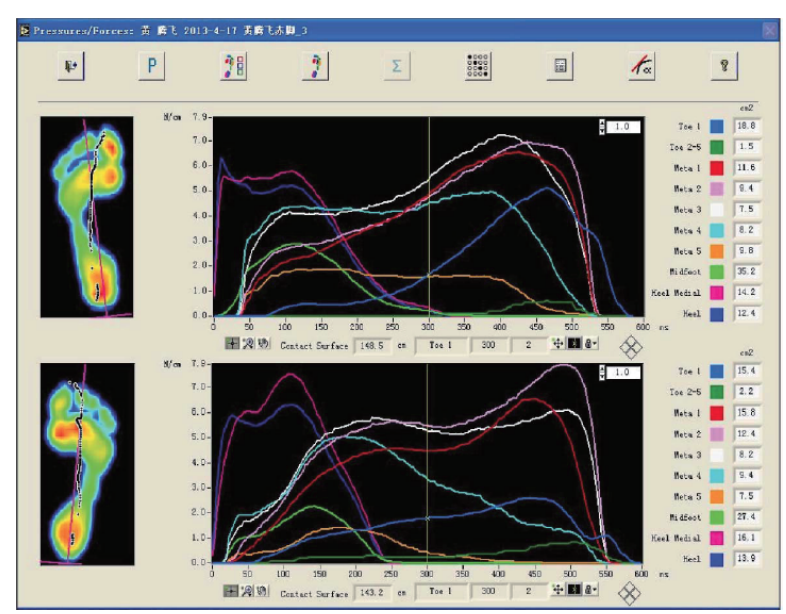

Fig. 3 The foot pressure distribution system

\section{Results and Discussion}

\subsection{Plantar Images Pre-processing}

To get a better fusion effect, initially, the plantar pressure is preprocessed by applying image filtering. To find the most suitable image enhancement method for the plantar pressure image, Gauss filtering, median filtering, and bilateral filtering are applied. The enhanced source (original) image using theses filters respectively are illustrated in Fig. 4. 


\subsection{Image Fusion using Wavelet Transform}

In this experiment, several types of wavelet basis function are used. The low frequency coefficient pixel averaging based on image fusion rules of maximum frequency coefficient of the fused image is obtained. A comparative analysis of the evaluation index is also introduced. In the experiment, initially, a decomposition layer is tested. As we know that the more layers of wavelet transform and the richer the frequency range of fusion, the more abundant the details of the fusion results. But the level of wavelet decomposition is much more, the quality of image fusion is not higher, because wavelet transform decomposition is a division of the band. Decomposition of more layers, the sub-band produce more frequency division and more detailed. The output signal on a frequency decomposition is the next level of input frequency. Decomposition means more filter layers increase between the greater the number of shift caused by information; on the other hand, wavelet transform to extension of the boundary layers distortion is greatly caused by boundary. Usually, it is feasible to choose $3-5$ layer based on wavelet. In this paper, different wavelet basis functions are also tested for image fusion as follows using the two source images in Fig.5.

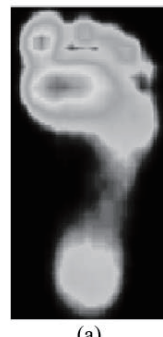

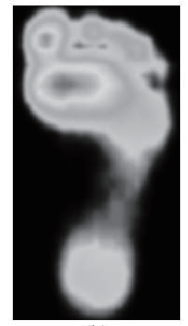

(b)

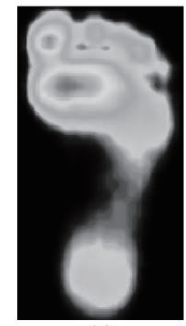

(c)

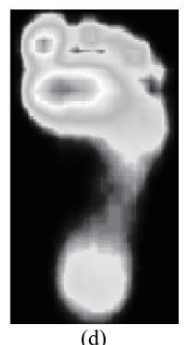

(b) Gauss filter (c) median filter (d) bilateral filter

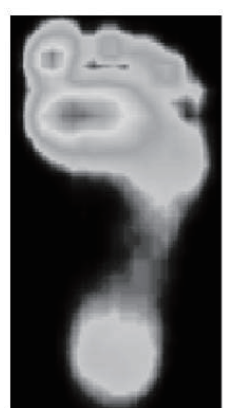

(a) source image 1

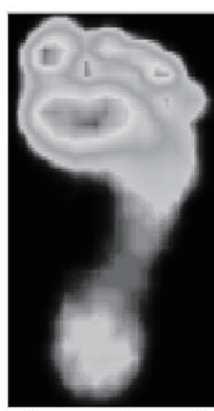

(b)source image 2

Fig. 5 The two source images 
Table 2 Evaluation indexes with 3 decomposition layers fusion.

\begin{tabular}{lllllll}
\hline & $\mathrm{M}$ & $\mathrm{SD}$ & $\mathrm{E}$ & $\mathrm{AG}$ & $\mathrm{SP}$ & $\mathrm{MCE}$ \\
\hline Source Image 1 & 87.5904 & 87.4752 & 5.8508 & 3.3749 & 10.5550 & - \\
Source Image 2 & 89.0931 & 87.4008 & 5.8405 & 3.4230 & 10.8142 & - \\
haar & 88.4255 & 86.8396 & 6.0939 & 5.1051 & 13.3824 & 0.1686 \\
db2 & 88.3655 & 86.2862 & 6.1214 & 4.6707 & 12.9493 & 0.1632 \\
sym4 & 88.3576 & 86.2201 & 6.1675 & 4.5838 & 12.8128 & 0.1651 \\
coif2 & 88.3486 & 86.1972 & 6.1233 & 4.5514 & 12.7613 & 0.1598 \\
bior5.5 & 88.4081 & 86.1959 & 6.1432 & 4.6346 & 13.0326 & 0.1696 \\
\hline
\end{tabular}

\subsubsection{Plantar Pressure Image Fusion using 3 Decomposition Layers}

Fig. 6 demonstrated the fussed images using different wavelet families for 3 decomposition layers each using the source images in Fig. 5. In addition, Table 2 reported the measured evaluation metrics with 3 decomposition layers fusion.

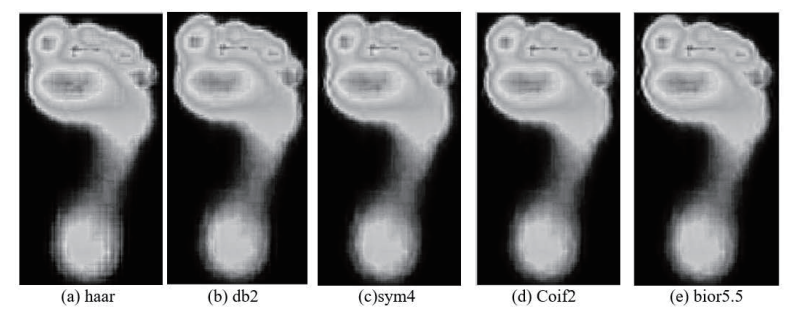

Fig. 6 Plantar pressure image fusion with 3 decomposition layers

Table 2 reported that haar wavelet with 3 levels decomposition outperformed all the other reported wavelet families in terms of all measured evaluation metrics as it achieved the highest mean, standard deviation, average gradient, and spatial frequency of $88.4255,86.8396,5.1051$, and 13.3824 value; respectively. In addition, it has the least information entropy of 6.0939 , while it gains cross entropy of 0.1686 .

\subsubsection{Plantar Pressure Image Fusion using 4 Decomposition Layers}

Fig. 7 demonstrated the fussed images of the source images in Fig. 5 using different wavelet families for 4 decomposition layers each. In addition, Table 3 reported the measured evaluation metrics with 4 decomposition layers fusion.

Table 3 reported that haar wavelet with 4 levels decomposition outperformed all the other reported wavelet families in terms of all measured evaluation metrics as it achieved the highest mean, standard deviation, average gradient, and spatial frequency of $88.8074,88.0532,5.3934$, and 14.2475 value; 
Table 3 Evaluation indexes with 4 decomposition layers fusion

\begin{tabular}{lllllll}
\hline & $\mathrm{M}$ & $\mathrm{SD}$ & $\mathrm{E}$ & $\mathrm{AG}$ & $\mathrm{SP}$ & $\mathrm{MCE}$ \\
\hline Source Image1 & 87.5904 & 87.4752 & 5.8508 & 3.3749 & 10.5550 & - \\
Source Image 2 & 89.0931 & 87.4008 & 5.8405 & 3.4230 & 10.8142 & - \\
haar & 88.8074 & 88.0532 & 6.0173 & 5.3934 & 14.2475 & 0.1901 \\
db2 & 88.4590 & 86.9650 & 6.1036 & 4.8875 & 13.3289 & 0.2017 \\
sym4 & 88.5190 & 86.9167 & 6.2727 & 4.8789 & 13.3038 & 0.2361 \\
coif2 & 88.5492 & 86.8369 & 6.2185 & 4.8228 & 13.2015 & 0.2264 \\
bior5.5 & 88.6019 & 86.8279 & 6.2710 & 4.9166 & 13.4766 & 0.2184 \\
\hline
\end{tabular}

respectively. In addition, it has the least information entropy of 6.0173 , while it gains the least cross entropy value of 0.1901 .

\subsubsection{Plantar Pressure Image Fusion using 5 Decomposition Layers}

Fig. 8 demonstrated the fussed images of the source images in Fig. 5 using different wavelet families for 5 decomposition layers each. In addition, Table 4 reported the measured evaluation metrics with 5 decomposition layers fusion.

Table 4 reported that haar wavelet with 5 levels decomposition outperformed all the other reported wavelet families in terms of all measured evaluation metrics as it achieved the highest mean, standard deviation, average gradient, and spatial frequency of $89.2817,89.4913,5.4196$, and 14.3364 value; respectively. In addition, it has the least information entropy of 5.9323 ,

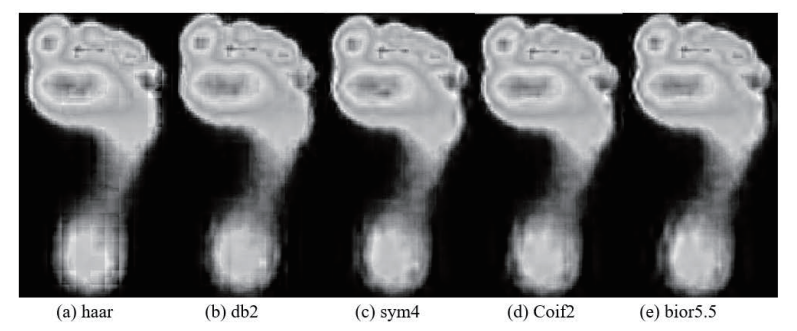

Fig. 7 Plantar pressure image fusion with 4 layer

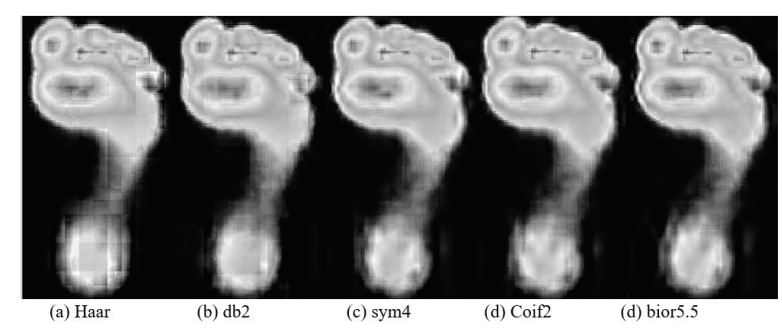

Fig. 8 Plantar pressure image fusion with 5 decomposition layers 
Table 4 Evaluation indexes with 5 decomposition layers fusion.

\begin{tabular}{lllllll}
\hline & $\mathrm{M}$ & $\mathrm{SD}$ & $\mathrm{E}$ & $\mathrm{AG}$ & $\mathrm{SP}$ & $\mathrm{MCE}$ \\
\hline Source Image 1 & 87.5904 & 87.4752 & 5.8508 & 3.3749 & 10.5550 & \\
Source Image 2 & 89.0931 & 87.4008 & 5.8405 & 3.4230 & 10.8142 & \\
haar & 89.2817 & 89.4913 & 5.9323 & 5.4196 & 14.3364 & 0.2206 \\
db2 & 89.2680 & 88.3459 & 6.2683 & 5.0845 & 13.5799 & 0.3117 \\
sym4 & 89.0541 & 87.7272 & 6.4195 & 5.0405 & 13.4819 & 0.3236 \\
coif2 & 89.1543 & 87.5695 & 6.3568 & 5.0065 & 13.3726 & 0.2972 \\
bior5.5 & 89.1880 & 87.5871 & 6.4163 & 5.1092 & 13.6579 & 0.2887 \\
\hline
\end{tabular}

while it gains the least cross entropy value of 0.2206 . Generally, the preceding reported evaluation results of $3,4,5$ decomposition layers based on wavelet basis functions- haar, DB2, sym4, coif2, and bior5.5; respectively in Tables 2 through 4 established that based on the information entropy, the wavelet transform image fusion with average gradient, spatial frequency and other indicators are superior to the original image. Furthermore, the results depicted that the entropy of the fused images is significantly increased compared to the source (original) image. In addition, the details of the image contrast, texture and clarity are greatly improved. Thus, the quality of image fusion is better compared to the source images. Generally, Tables 2 through 4 illustrated that the values of wavelet bases haar and bior5.5 were 88.4255 and 88.4081 , and the remaining mean values are close to 88.348688 .3655 . The maximum and minimum values of cross entropy are 0.1696 and 0.1598 ; respectively, and the amplitude is only about 0.0098 . In order to be more intuitive to illustrate the different methods of image fusion of each evaluation index, the maximum value of each evaluation index is normalized as shown in Fig. 9. The x-axis in the figure is the type of wavelet basis of haar, db2, sym4, coif2, and bior5.5. The $\mathrm{y}$-axis represents the normalized mean value, standard deviation, information entropy, average gradient, spatial frequency, and average cross entropy. Table 5 demonstrated that the decomposition of 3,4 and 5 based on the fusion of haar wavelet image achieved the largest mean, standard deviation, average gradient, and spatial frequency values compared to the other basis functions. Moreover, the haar wavelet function of the MCE with five layers decomposition has the minimum description of haar wavelet based image fusion, and the difference between images is very small and has high clarity. The information entropy of the fused image based on the wavelet basis function sym 4 is the largest, which shows that the information of the fused image based on sym4 is the most, and the information contained in the fused image is abundant. Generally, based on the above analysis, the image fusion methods based on wavelet basis functions haar and sym4 have no significant difference in the fusion of the plantar pressure images.

Moreover, to study different wavelet functions on plantar pressure image fusion effect, the image information on the image features and the various evaluation indexes are normalized. Weight index is to determine the corresponding weight coefficient according to its importance in the evaluation. The weights 
Table 5 Normalized evaluation indexes.

\begin{tabular}{llllllll}
\hline M & \multicolumn{7}{c}{ AG } \\
& 3 layer & 4 layer & 5 layer & & 3 layer & 4 layer & 5 layer \\
haar & 1.0000 & 1.0000 & 1.0000 & haar & 1.000 & 1.0000 & 1.0000 \\
db2 & 0.9993 & 0.9961 & 0.9998 & db2 & 0.9149 & 0.9062 & 0.9382 \\
sym4 & 0.9992 & 0.9958 & 0.9975 & sym4 & 0.8979 & 0.9046 & 0.9301 \\
coif2 & 0.9991 & 0.9971 & 0.9986 & coif2 & 0.8915 & 0.8942 & 0.9238 \\
bior5.5 & 0.9998 & 0.9977 & 0.9990 & bior5.5 & 0.9078 & 0.9115 & 0.9427 \\
\hline SD & & & & SF & & & \\
& & & & & 3 layer & 4 layer & 5 layer \\
haar & 1.0000 & 1.0000 & 1.0000 & haar & 1.0000 & 1.000 & 1.0000 \\
db2 & 0.9936 & 0.9876 & 0.9872 & db2 & 0.9676 & 0.9355 & 0.9472 \\
sym4 & 0.9929 & 0.9871 & 0.9803 & sym4 & 0.9574 & 0.9338 & 0.9404 \\
coif2 & 0.9926 & 0.9862 & 0.9785 & coif2 & 0.9536 & 0.9366 & 0.9328 \\
bior5.5 & 0.9926 & 0.9861 & 0.9787 & bior5.5 & 0.9739 & 0.9459 & 0.9527 \\
\hline E & & & & MCE & & & \\
\hline & & & & & & \\
haar & 0.988 & 0.9593 & 0.9241 & haar & 0.9941 & 0.8052 & 0.6817 \\
db2 & 0.9925 & 0.973 & 0.9764 & db2 & 0.9623 & 0.8543 & 0.9632 \\
sym4 & 1.0000 & 1.0000 & 1.0000 & sym4 & 0.9735 & 1.0000 & 1.0000 \\
coif2 & 0.9928 & 0.9914 & 0.9902 & coif2 & 0.9422 & 0.9589 & 0.9184 \\
bior5.5 & 0.9961 & 0.9997 & 0.9995 & bior5.5 & 1.0000 & 0.9647 & 0.8922 \\
\hline
\end{tabular}

can be selected manually according to those six indexes in the comprehensive evaluation process. Information entropy is an important indicator to measure the information richness of the image, the average gradient reflects the clarity of the image; for the fused image of plantar pressure, the purpose is to get more information, so in accordance with the evaluation methods of image quality, according to the various indicators of the emphasis on the practical application of plantar pressure of the image, on the six indexes set the corresponding weights. The weights of each evaluation index were $10 \%, 10 \%, 30 \%$, $30 \%, 10 \%, 10 \%$ of $\mathrm{M}, \mathrm{SD}, \mathrm{E}, \mathrm{AG}, \mathrm{SF}$ and MCE respectively. Since the M$\mathrm{CE}$ is negative indicator, inverse normalization is used. Afterward, each index weighted sum is calculated, in addition, evaluation index weighting of different wavelet functions and values are compared as follows:

$$
\begin{aligned}
& 3 \text { layers: } \mathrm{SUM}(\text { haar })>\mathrm{SUM}(\text { bior5.5) }>\mathrm{SUM}(\mathrm{db} 2)>\mathrm{SUM}(\operatorname{sym} 4)>\mathrm{SUM}(\text { coif2 }) \\
& 4 \text { layers: } \mathrm{SUM}(\text { haar })>\mathrm{SUM}(\mathrm{db} 2)>\mathrm{SUM}(\text { bior} 5.5)>\mathrm{SUM}(\operatorname{sym} 4)>\mathrm{SUM}(\text { coif2 }) \\
& 5 \text { layers: } \mathrm{SUM}(\text { haar })>\mathrm{SUM}(\text { bior} 5.5)>\mathrm{SUM}(\text { coif2 })>\mathrm{SUM}(\mathrm{db} 2)>\mathrm{SUM}(\operatorname{sym} 4)
\end{aligned}
$$

Furthermore, haar wavelet transform achieved that SUM (5 layers) >SUM (4 layers) >SUM (3layers). These evaluation indexes normalization with 3,4 and 5 decomposition layers are reported in Tables 6 through 8 respectively.

Tables 6,7 ,and 8 , established that the weighted sum of the haar wavelet basis function is the best with 5-layer decomposition. 
Table 6 Evaluation indexes normalization with 3 decomposition layers.

\begin{tabular}{llllllll}
\hline & $\mathrm{M}$ & $\mathrm{SD}$ & $\mathrm{E}$ & $\mathrm{AG}$ & $\mathrm{SP}$ & $\mathrm{MCE}$ & SUM \\
\hline - & $10 \%$ & $10 \%$ & $30 \%$ & $30 \%$ & $10 \%$ & $10 \%$ & - \\
haar & 1.0000 & 1.0000 & 0.9880 & 1.0000 & 1.0000 & 0.9941 & 0.8970 \\
db2 & 0.9993 & 0.9936 & 0.9925 & 0.9149 & 0.9676 & 0.9623 & 0.8720 \\
sym4 & 0.9992 & 0.9929 & 1.0000 & 0.8979 & 0.9574 & 0.9735 & 0.8670 \\
coif2 & 0.9991 & 0.9926 & 0.9928 & 0.8915 & 0.9536 & 0.9422 & 0.8656 \\
bior5.5 & 0.9998 & 0.9926 & 0.9961 & 0.9078 & 0.9739 & 1.0000 & 0.8678 \\
\hline
\end{tabular}

Table 7 Evaluation indexes normalization with 4 decomposition layers.

\begin{tabular}{llllllll}
\hline & $\mathrm{M}$ & $\mathrm{SD}$ & $\mathrm{E}$ & $\mathrm{AG}$ & $\mathrm{SP}$ & $\mathrm{MCE}$ & $\mathrm{SUM}$ \\
\hline - & $10 \%$ & $10 \%$ & $30 \%$ & $30 \%$ & $10 \%$ & $10 \%$ & - \\
haar & 1.0000 & 1.0000 & 0.9593 & 1.0000 & 1.0000 & 0.8052 & 0.9073 \\
db2 & 0.9961 & 0.9876 & 0.9730 & 0.9062 & 0.9355 & 0.8543 & 0.8703 \\
sym4 & 0.9958 & 0.9871 & 1.0000 & 0.9046 & 0.9338 & 1.0000 & 0.8625 \\
coif2 & 0.9971 & 0.9862 & 0.9914 & 0.8942 & 0.9366 & 0.9589 & 0.8168 \\
bior5.5 & 0.9977 & 0.9861 & 0.9997 & 0.9115 & 0.9459 & 0.9647 & 0.8738 \\
\hline
\end{tabular}

Table 8 Evaluation indexes normalization with 5 decomposition layers.

\begin{tabular}{llllllll}
\hline & $\mathrm{M}$ & $\mathrm{SD}$ & $\mathrm{E}$ & $\mathrm{AG}$ & $\mathrm{SP}$ & $\mathrm{MCE}$ & $\mathrm{SUM}$ \\
\hline - & $10 \%$ & $10 \%$ & $30 \%$ & $30 \%$ & $10 \%$ & $10 \%$ & - \\
haar & 1.0000 & 1.0000 & 0.9241 & 1.0000 & 1.0000 & 0.6817 & 0.9091 \\
db2 & 0.9998 & 0.9872 & 0.9764 & 0.9382 & 0.9472 & 0.9632 & 0.8715 \\
sym4 & 0.9975 & 0.9803 & 1.0000 & 0.9301 & 0.9404 & 1.0000 & 0.8709 \\
coif2 & 0.9986 & 0.9785 & 0.9902 & 0.9238 & 0.9328 & 0.9184 & 0.8734 \\
bior5.5 & 0.9990 & 0.9787 & 0.9995 & 0.9427 & 0.9527 & 0.8922 & 0.8865 \\
\hline
\end{tabular}

3.3 Plantar Image fusion using Laplace Pyramid Transform

Fig. 9 illustrated the plantar images fusion of the two source images in Fig.5 with different Laplacian Pyramid layers of values 3, 4 and 5. In addition, Table 8 reported the evaluation indexes using Laplacian pyramid with different decomposition layers.

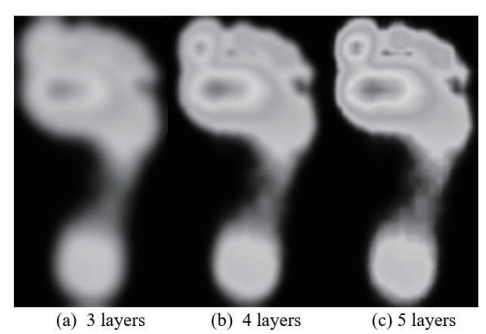

Fig. 9 Fusion with different layer Laplacian pyramid 
Table 9 Evaluation indexes normalization with 5 decomposition layers.

\begin{tabular}{lllllll}
\hline & M & SD & E & AG & SP & MCE \\
\hline Source Image 1 & 87.5904 & 87.4752 & 5.8508 & 3.3749 & 10.5550 & \\
Source Image 2 & 89.0931 & 87.4008 & 5.8405 & 3.4230 & 10.8142 & \\
3 layers & 187.0981 & 99.2637 & 3.3603 & 1.4375 & 30.3257 & 1.2730 \\
4 layers & 187.1093 & 98.6133 & 3.4035 & 1.3988 & 30.0551 & 1.2450 \\
5 layers & 187.1709 & 97.0241 & 3.5305 & 1.3142 & 29.5264 & 1.3193 \\
\hline
\end{tabular}

Table 9 indicated that the information entropy and average gradient value using Laplacian Pyramid at different layers achieved smaller values compared to that the source images have. This result established that the fused image has information loss compared to the source image. Furthermore, the blurred fused image especially with the 3 levels decomposition indicated that the image fusion based on Laplace Pyramid transform is poor. Consequently, the haar wavelet basis function of the foot pressure image fusion performs the best effective fusion with 5 levels decomposition, indicating that higher level of wavelet transforms using haar basis function yields outperformed quality fused images compared to the other functions reported in the current study. Accordingly, it is recommended to use optimization algorithm to select the optimal decomposition level as well as the optimal basis wavelet function. Moreover, further improvement on the quality of image fusion and evaluation of the reliability of the results need to be considered in future works.

\section{Conclusion}

The present work focused on plantar pressure image fusion technologies. A comparative study of using Laplace pyramid transform and fused images with haar wavelet at different decomposition levels have been studied. In addition, the analysis depicted the superiority of the haar wavelet at different decomposition layers compared to db2, db2, sym4, coif2 and bior5.5. Generally, the Haar wavelet transform was applied and recommended to the plantar pressure of image fusion based on the objective evaluation of image quality. The results indicated that image haar wavelet fusion has achieved superior effect on the fusion quality as well as improved the image entropy. It provided plantar pressure level support to improve the image clarity, reliability, and provided a strong guarantee for the further research. The evaluation indexes of M, SD, $\mathrm{E}, \mathrm{AG}, \mathrm{SF}$, and MCE were used for evaluating the fused images.

\section{References}

1. Z. Li, N. Dey, A. S. Ashour, L. Cao, Y. Wang, D. Wang, P. McCauley, V. E. Balas, K. Shi, and F. Shi, Convolutional neural network based clustering and manifold learning method for diabetic plantar pressure imaging dataset, J. Med. Imag. and Health Inform., 7, 1-14, 2017. 
2. D. Wang, Z. Li, L. Cao, V. E Balas., N. Dey., A. S. Ashour., P. McCauley, and F. Shi, Multi-scale plantar pressure imaging data fusion incorporating improved Gaussian mixture operator and fuzzy weighted evaluation system, IEEE Sensors J., 17, (5): 1407$1420,2017$.

3. Z. Li, V. E. Balas, P. McCauley, and F. Shi, Multi-source Information Fusion Model in Rule-based Fuzzy Inference System incorporating Gaussian Density Function, J. Intel. E Fuzzy Syst., 29 2335-2344, 2015.

4. D. Wang, Z. Li, N. Dey, A. S. Ashour, R. S. Sherratt and F. Shi, Case-Based Reasoning for Product Style Construction and Fuzzy Analytic Hierarchy Process Evaluation Modeling Using Consumers Linguistic Variables, IEEE Access, 5 4900-4912, 2017.

5. P. E. Chatzistergos, R. Naemi, N. Chockalingam, An MRI compatible loading device for the reconstruction of clinically relevant plantar pressure distributions and loading scenarios of the forefoot, Med. Eng. Phy. 36 (9): 1205-1211, 2014.

6. N.L.W. Keijsers, N.M. Stolwijk, J.W.K. Louwerens, J. Duysens, Classification of forefoot pain based on plantar pressure measurements, Clin. Biome 28 (3): 350-356, 2013.

7. K. Deschamps, F. Staes, D. Desmet, P. Roosen, G.A. Matricali, N. Keijsers, F. Nobels, J. Tits, H. Bruyninckx, A color-code based method for the interpretation of plantar pressure measurements in clinical gait analysis, Gait Posture 41 (3): 852-856, 2015.

8. C. J. Bennetts, T. M. Owings, A. Erdemir, G. Botek, P. R. Cavanagh, Clustering and classification of regional peak plantar pressures of diabetic feet, J. Biome 46 (1): 19-25, 2013.

9. H.M. A. Angari, A. H. Khandoker, S. Lee, W. Almahmeed, H. S. A. Safar, H. F. Jelinek, K. Khalaf, Novel dynamic peak and distribution plantar pressure measures on diabetic patients during walking, Gait Posture 51, 261-267, 2017.

10. W. -M. Chen, S.-J. Lee, P. V. S. Lee, Plantar pressure relief under the metatarsal heads - Therapeutic insole design using three-dimensional finite element model of the foot, $J$. Biomech. 48 (4): 659-665, 2015.

11. L Moraru, D Bibicu, A Biswas, Standalone functional CAD system for multi-object case analysis in hepatic disorders, Computers in Biology and Medicine, 43, 967-974, 2013

12. B Bhosale, L Moraru, B S. Ahmed, D Riser, A Biswas, Multi-resolution analysis of wavelet like soliton solution of KDY equation, Proceedings of the Romanian academy, Series A, 15 (1): 18-26, 2014

13. Z. Wang, C. Gong, A multi-faceted adaptive image fusion algorithm using a multiwavelet-based matching measure in the PCNN domain, Applied Soft Computing, 2017, http://dx.doi.org/10.1016/j.asoc.2017.02.03519, Accessed March 2017

14. L. Piras, G. Giacinto, Information fusion in content based image retrieval: A comprehensive overview, Inform Fusion 37, 50-60, 2017.

15. J. Zong, T. Qiu, Medical image fusion based on sparse representation of classified image patches, Biomedi Signal Proces 34, 195-205, 2017.

16. T. P. Frye, A. K. George, A. Kilchevsky, M. Maruf, M. M. Siddiqui, M. Kongnyuy, A. Muthigi, H. Han, H. L. Parnes, M. Merino, P. L. Choyke, B. Turkbey, B. Wood, P. A. Pinto, Magnetic Resonance Imaging-Transrectal Ultrasound Guided Fusion Biopsy to Detect Progression in Patients with Existing Lesions on Active Surveillance for Low and Intermediate Risk Prostate Cancer, J. Urol. 197(3): 640-646, 2017.

17. M. Valerio, T. T. Shah, P. Shah, N. Mccartan, M. Emberton, M. Arya, H. U. Ahmed, Magnetic resonance imaging-transrectal ultrasound fusion focal cryotherapy of the prostate: A prospective development study, Urol Oncol 35(4): 150.e1-150.e7, 2017

18. Y. Zhang, X. Bai, T. Wang, Boundary finding based multi-focus image fusion through multi-scale morphological focus-measure, Inform Fusion 35, 81-101, 2017.

19. C. He, J. Shao, X. Xu, D. Ouyang, L. Gao, Exploiting score distribution for heterogenous feature fusion in image classification, Neurocomputing, 2017 http://dx.doi.org/10.1016/j.neucom.2016.09.129, Accessed April 2017.

20. Y. Liu, X. Chen, H. Peng, Z. Wang, Multi-focus image fusion with a deep convolutional neural network, Inform. Fusion 36, 191-207, 2017.

21. S. Tedmori, N. A. Najdawi, Image cryptographic algorithm based on the Haar wavelet transform, Inform Sci 269, 21-34, 2014.

22. S. S. Ray, A. Patra, Haar wavelet operational methods for the numerical solutions of fractional order nonlinear oscillatory Van der Pol system, Appl. Math. Comp. 220, 659667,2013 
23. K. A. Bhandari, R. M. Ramchandra, An Innovative Remote Sensing Image Retrieval Techniques Based on Haar Wavelet-LTRP and ANFIS, Proc. Comp. Sci. 79, 391-401, 2016.

24. B. Mohamed, A. Issam, A. Mohamed, B. Abdellatif, ECG Image Classification in Real time based on the Haar-like Features and Artificial Neural Networks, Proc. Comp. Sci. 73, 32-39, 2015.

25. Z. Chang, X. Ban, and Y. Wang, Fatigue driving detection based on Haar feature and extreme learning machine, J. China Uni. Posts Telecomm. 23 (4) : 91-100, 2016.

26. H. J. O. Domnguez, L. O. Mynez, O. O. V. Villegas, B. Mederos, J. M. Meja, V. G. C. Snchez, Denoising of high resolution small animal 3D PET data using the non-subsampled Haar wavelet transform, Nuclear Instruments and Methods in Physics Research Section A: Accelerators, Spectrometers, Dete. Ass. Equipt 784, 581-584, 2015.

27. A. Koc, B. Bartan, E. Gundogdu, T. cukur, H. M. Ozaktas, Sparse representation of two- and three-dimensional images with fractional Fourier, Hartley, linear canonical, and Haar wavelet transforms, Exp. Syst. Appl. 77, 247-255, 2017.

28. A. Banerjee, A. Dutta, Performance Comparison of Cosine, Haar, Walsh-hadamard, Fourier and Wavelet Transform for Shape based Image Retrieval Using Fuzzy Similarity Measure, Proc. Tech. 10, 623-627, 2013.

29. Sarwar Kamal, Linkon Chowdhury, Mohammad Ibrahim Khan, Amira S. Ashour, Jo?o Manuel R.S. Tavares, Nilanjan Dey, Hidden Markov Model and Chapman Kolmogrov for Protein Structures Prediction from Images, Computational Biology and Chemistry 68, 231-244, 2017

30. Sarwar Kamal, Nilanjan Dey, Sonia Farhana Nimmy, Shamim H Ripon, Nawab Yousuf Ali, Amira Salah Ashour, Wahiba Ben Abdessalem Karaa and Fuqian Shi, Evolutionary Framework for Coding Area Selection from Cancer Data, Neural Computing and Applications 29 (4):1015-1037, 2018

31. Sarwar Kamal, Shamim H Ripon, Nilanjan Dey, Amira S. Ashour, V. Santhi, A MapReduce Approach to Diminish Imbalance Parameters for Big Deoxyribonucleic Acid Dataset, Computer Methods and Programs in Biomedicine 131, 191-206, 2016

32. Sarwar Kamal, Sazia Parvin, Amira S. Ashour, Fuqian Shi, Nilanjan Dey, De-Bruijn graph with MapReduce framework towards metagenomic data classification, International Journal of Information Technology 9, 59-75, 2017

33. S.G. Mallat, Z. Zhang, A theory for multiresolution signal decomposition: the wavelet representation, IEEE Trans. Pattern Anal 11, 674-691, 1989.

34. D. Oliva, S. Hinojosa, E. Cuevas, G. Pajares, O. Avalos, J. Glvez, Cross entropy based thresholding for magnetic resonance brain images using Crow Search Algorithm, Exp. Syst. Appl. 79, 164-180, 2017.

35. J. Qian, J. Yang, Y. Tai, H. Zheng, Exploring deep gradient information for biometric image feature representation, Neurocomputing 213, 162-171, 2016.

36. W. Zhou, L. Yu, W. Qiu, Y. Zhou, M. Wu, Local gradient patterns (LGP): An effective local-statistical-feature extraction scheme for no-reference image quality assessment, Inform. Sci. 397-398 1-14, 2017. 